Acta Crystallographica Section D

Biological

Crystallography

ISSN 0907-4449

\title{
Structural basis of high-order oligomerization of the cullin-3 adaptor SPOP
}

\author{
Laura K. van Geersdaele, Mark A. Stead, Christopher M. Harrison, \\ Stephen B. Carr, Helen J. Close, Gareth O. Rosbrook, Simon D. Connell \\ and Stephanie C. Wright
}

Acta Cryst. (2013). D69, 1677-1684

Copyright (C) International Union of Crystallography

Author(s) of this paper may load this reprint on their own web site or institutional repository provided that this cover page is retained. Republication of this article or its storage in electronic databases other than as specified above is not permitted without prior permission in writing from the IUCr.

For further information see http://journals.iucr.org/services/authorrights.html

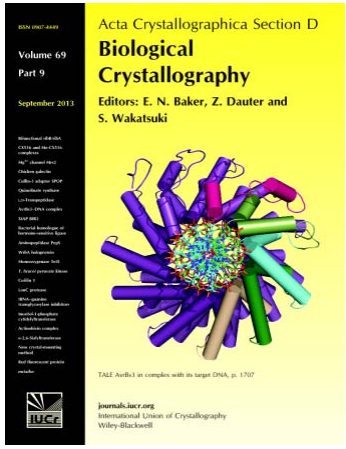

\begin{abstract}
Acta Crystallographica Section D: Biological Crystallography welcomes the submission of papers covering any aspect of structural biology, with a particular emphasis on the structures of biological macromolecules and the methods used to determine them. Reports on new protein structures are particularly encouraged, as are structure-function papers that could include crystallographic binding studies, or structural analysis of mutants or other modified forms of a known protein structure. The key criterion is that such papers should present new insights into biology, chemistry or structure. Papers on crystallographic methods should be oriented towards biological crystallography, and may include new approaches to any aspect of structure determination or analysis. Papers on the crystallization of biological molecules will be accepted providing that these focus on new methods or other features that are of general importance or applicability.
\end{abstract}

Crystallography Journals Online is available from journals.iucr.org 
Acta Crystallographica Section D

Biological

Crystallography

ISSN 0907-4449

Laura K. van Geersdaele, ${ }^{\mathbf{a}} \neq$

Mark A. Stead, ${ }^{a} \ddagger$ Christopher $M$.

Harrison, ${ }^{a}$ Stephen B. Carr,

Helen J. Close, ${ }^{a}$ Gareth $O$.

Rosbrook, $^{\text {a Simon D. Connell }}$

and Stephanie C. Wright ${ }^{\mathrm{a} *}$

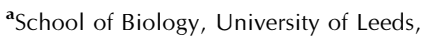
Leeds LS2 9JT, England, 'besearch Complex at Harwell, Rutherford Appleton Laboratory, Harwell Oxford, Didcot OX11 OFA, England, and ${ }^{\mathbf{C}}$ School of Physics and Astronomy,

University of Leeds, Leeds LS2 9JT, England

₹ LKvG and MAS contributed equally to this work.

Correspondence e-mail: s.c.wright@leeds.ac.uk

\section{Structural basis of high-order oligomerization of the cullin-3 adaptor SPOP}

Protein ubiquitination in eukaryotic cells is mediated by diverse E3 ligase enzymes that each target specific substrates. The cullin E3 ligase complexes are the most abundant class of E3 ligases; they contain various cullin components that serve as scaffolds for interaction with substrate-recruiting adaptor proteins. SPOP is a BTB-domain adaptor of the cullin-3 E3 ligase complexes; it selectively recruits substrates via its $\mathrm{N}$-terminal MATH domain, whereas its BTB domain mediates dimerization and interactions with cullin-3. It has recently been recognized that the high-order oligomerization of SPOP enhances the ubiquitination of substrates. Here, a dimerization interface in the SPOP C-terminus is identified and it is shown that the dimerization interfaces of the BTB domain and of the C-terminus act independently and in tandem to generate high-order SPOP oligomers. The crystal structure of the dimeric SPOP C-terminal domain is reported at $1.5 \AA$ resolution and it is shown that Tyr353 plays a critical role in high-order oligomerization. A model of the high-order SPOP oligomer is presented that depicts a helical organization that could enhance the efficiency of substrate ubiquitination.

\section{Introduction}

Ubiquitination is a highly regulated post-translational modification that targets proteins for degradation by the $26 \mathrm{~S}$ proteasome and also plays nonproteolytic roles in controlling protein-protein interactions, subcellular localization and enzymatic activity (reviewed in Komander \& Rape, 2012). It is carried out by an enzyme cascade in which ubiquitin is first transferred from an E1 activating enzyme onto an E2 conjugating enzyme. An E3 ubiquitin-protein ligase then catalyses the transfer of ubiquitin from the E2 enzyme to the substrate. E3 ligases of the HECT (homologous to the E6AP carboxyl terminus) domain family contain an active-site cysteine that forms an obligate thioester intermediate with ubiquitin; in contrast, the RING (really interesting new gene) finger E3 ligases do not form a catalytic intermediate with ubiquitin, but instead act to bring the substrate and E2 enzyme into close proximity to facilitate ubiquitin transfer. There are approximately 30 HECT-domain E3s and over 600 RING-finger E3s in mammals, and this diversity allows the independent ubiquitination of different substrates. The regulated ubiquitination of specific proteins is critical for fundamental biological processes such as the cell cycle and apoptosis, and alterations in ubiquitination pathways have been implicated in many human disease states (reviewed in Nalepa et al., 2006; Petroski, 2008).

The cullin-RING E3 ligase complexes are multisubunit enzymes that together constitute the largest subclass of RING-finger E3 ligases. They contain a cullin component
Received 25 March 2013

Accepted 8 May 2013

PDB References: SPOP ${ }^{270-374}$ L273D L282D L285K, 4hs2 ; SPOP $^{169-374 \text { Y353E }}, 4 j 8 z$
(C) 2013 International Union of Crystallography Printed in Singapore - all rights reserved 
(Cul1, Cul2, Cul3, Cul4a, Cul4b, Cul5 or Cul7) together with various adaptor modules that selectively recognize specific substrates. The N-termini of the cullins interact with the adaptors, whereas their C-termini interact with a RINGdomain protein that in turn recruits the E2 enzyme (reviewed in Zimmerman et al., 2010). The Cul3 adaptors (reviewed in Pintard et al., 2004) recruit substrates via domains such as Kelch or MATH (mephrin and TRAF homology), and they interact with cullin via their BTB [bric-à-brac, tramtrack and broad complex; also known as POZ (poxvirus and zinc finger)] domain, a versatile protein-protein interaction domain that is also found in zinc-finger transcription factors (reviewed in Stogios et al., 2005).

SPOP (speckle-type POZ protein) is a MATH-BTB-type Cul3 adaptor that regulates cell proliferation and development (Nagai et al., 1997). The MATH domain of SPOP interacts with serine/threonine-rich degrons in target proteins (Zhang et al., 2009; Zhuang et al., 2009) that include the apoptotic regulator Daxx (Kwon et al., 2006), the steroid receptor co-activator SRC-3 (Li et al., 2011) and the variant histone protein MacroH2A (Takahashi et al., 2002). SPOP has been implicated both as an oncogene (Liu et al., 2009; Kim et al., 2011) and as a tumour suppressor (Barbieri et al., 2012; Li et al., 2011) in human cancer.

Substrate ubiquitination is enhanced by the self-association of various E3 ligase components (reviewed in Zimmerman et al., 2010), and structures of parts of the Cul3 adaptors SPOP and gigaxonin (Zhuang et al., 2009) revealed BTB-mediated dimers that resemble the BTB-domain dimers found in transcription factors. Many SPOP substrates contain multiple weak degrons that may simultaneously bind to the two MATH domains of an SPOP dimer (Zhang et al., 2009; Zhuang et al., 2009), thereby enhancing ubiquitination. Although the dimerization properties of BTB domains have been well characterized, it is recognized that higher-order oligomerization is important for the activities of several BTB proteins (see, for example, Espinás et al., 1999), and it has recently been shown that the high-order oligomerization of SPOP leads to enhanced substrate ubiquitination by Cul3 E3 ligase complexes (Errington et al., 2012); the structural basis of these important high-order associations is unknown. Here, we identify a novel dimerization interface in the C-terminus of SPOP that, in concert with the BTB domain, directs the formation of high-order oligomers. The X-ray crystal structure of the SPOP C-terminal domain enables us to propose a model of the high-order SPOP oligomer that reveals the mechanism by which substrate ubiquitination is enhanced by adaptor oligomerization.

\section{Materials and methods}

\subsection{Cloning}

A cDNA encoding human SPOP residues 1-374 was amplified by PCR from a human placental cDNA library. SPOP fragments were cloned into pGEX-6P-1 (GE Healthcare) for bacterial expression of GST-fusion proteins. Point mutations and deletions were generated by PCR using Phusion High-Fidelity DNA polymerase (Thermo Scientific). The SPOP proteins expressed in bacteria were SPOP ${ }^{169-374}$, $\mathrm{SPOP}^{169-334}, \mathrm{SPOP}^{169-296}, \mathrm{SPOP}^{169-359}, \mathrm{SPOP}^{169-355}, \mathrm{SPOP}^{169-344}$, SPOP $^{169-374 ~ Y 353 E}$ and SPOP ${ }^{270-374 ~ L 273 D ~ L 282 D ~ L 285 K . ~}$

\subsection{Protein expression and purification}

GST-SPOP proteins were expressed in Escherichia coli BL21 (DE3) pLysS. Recombinant proteins were induced with $0.1 \mathrm{~m} M$ IPTG at $289 \mathrm{~K}$. Bacteria were lysed using a cell disrupter (Constant Systems), the fusion proteins were bound to glutathione-Sepharose 4B (GE Healthcare) and the N-terminal GST tag was removed by cleavage with PreScission protease in $20 \mathrm{~m} M$ Tris- $\mathrm{HCl}, 75 \mathrm{~m} M \mathrm{NaCl}, 5 \mathrm{~m} M$ DTT $\mathrm{pH}$ 7.5. SPOP proteins were further purified by size-exclusion chromatography on a Superdex 75 HiLoad 26/60 column (GE Healthcare) in $20 \mathrm{~m} M$ Tris- $\mathrm{HCl}, 150 \mathrm{~m} M \mathrm{NaCl}, 5 \mathrm{~m} M$ DTT, $5 \%$ glycerol $\mathrm{pH}$ 8.6. Protein preparations for crystallization were concentrated using Vivaspin centrifugal concentrators (Sartorius). Selenomethionine-substituted GST-SPOP was expressed in E. coli B834 (DE3) pLysS; the cells were cultured in minimal medium in which the methionine was substituted by $45 \mu \mathrm{g} \mathrm{ml}^{-1}$ L-selenomethionine.

\subsection{Crystallization}

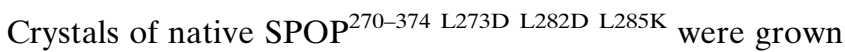
at $291 \mathrm{~K}$ by hanging-drop vapour diffusion against $26 \%$ PEG 1500, $0.1 M$ imidazole $\mathrm{pH}$ 6.5, $0.1 \mathrm{M} \mathrm{CaCl}_{2}, 7 \%$ 2-propanol; drops were prepared by mixing $2 \mu$ reservoir solution with $1 \mu \mathrm{l} 8 \mathrm{mg} \mathrm{ml}^{-1}$ protein solution. Crystals grew to average dimensions of $150 \times 25 \times 25 \mu \mathrm{m}$ within $5 \mathrm{~d}$. Crystals of selenomethionine-substituted SPOP ${ }^{270-374 ~ L 273 D ~ L 282 D ~ L 285 K ~ w e r e ~}$ grown by hanging-drop vapour diffusion against 29\% PEG 1500, 0.1 $M$ imidazole pH 6.5, $0.1 \mathrm{M} \mathrm{CaCl}_{2}, 7 \%$ 2-propanol. Crystals of SPOP ${ }^{169-374 ~ Y 353 E}$ were grown at $291 \mathrm{~K}$ by sittingdrop vapour diffusion against $32 \%$ PEG 3350, $17 \%$ 2-propanol, $0.1 M$ Tris- $\mathrm{HCl} \mathrm{pH} 8.5$; drops were prepared by mixing $2 \mu \mathrm{l}$ reservoir solution with $2 \mu 16 \mathrm{mg} \mathrm{ml}^{-1}$ protein solution and crystals grew to average dimensions of $200 \times 200$ $\times 15 \mu \mathrm{m}$ within $2 \mathrm{~d}$. Crystals of SPOP ${ }^{270-374 ~ L 273 D ~ L 282 D ~ L 285 K ~}$ were cryoprotected by transfer for $30 \mathrm{~s}$ into mother liquor supplemented with $20 \%$ glycerol before being flash-cooled in liquid nitrogen; crystals of SPOP ${ }^{169-374}$ Y353E were cryoprotected with mother liquor supplemented with $19 \%$ ethylene glycol.

\subsection{X-ray data collection and structure determination}

X-ray data were collected on Diamond Light Source beamlines I02 (native SPOP ${ }^{270-374}$ L273D L282D L285K), I04-1 (selenomethionine-substituted SPOP ${ }^{270-374 ~ L 273 D ~ L 282 D ~ L 285 K) ~}$ and I03 (native SPOP ${ }^{169-374 ~ Y 353 E}$ ). X-ray data were processed, reduced and scaled using $X D S$ (Kabsch, 2010) and SCALA (Evans, 2006) or AIMLESS using the xia2 pipeline (Winter, 2010). The structure of SPOP $^{270-374}$ L273D L282D L285K was determined by SAD phasing using SHELXD (Sheldrick, 2008; Schneider \& Sheldrick, 2002) for the heavy-atom 
search and SHELXE for initial phase estimation and density modification. The phases were combined with native amplitudes and an initial model was built using ARP/wARP (Cohen et al., 2008). The structure of SPOP ${ }^{169-374} \mathrm{Y} 353 \mathrm{E}$ was solved by molecular replacement using Phaser (McCoy et al., 2007) with the human SPOP BTB domain (PDB entry $3 \mathrm{htm}$; chain $A$ residues 177-299; Zhuang et al., 2009) and human SPOP CTD (structure of SPOP ${ }^{270-374}$ L273D L282D L285K ; residues 300-359) as independent search models. The model was built using ARP/wARP (Cohen et al., 2008) and underwent density modification using Parrot (Zhang et al., 1997). The models of SPOP $^{270-374 ~ L 273 D ~ L 282 D ~ L 285 K ~ a n d ~ S P O P ~}{ }^{169-374}$ Y353E $^{\text {underwent }}$ several rounds of iterative refinement in REFMAC5 (Murshudov et al., 2011) and model building using Coot (Emsley et al., 2010). The final models consist of SPOP residues 296-359 (SPOP ${ }^{270-374 ~ L 273 D ~ L 282 D ~ L 285 K) ~ a n d ~ 178-356 ~}$ $\left(\mathrm{SPOP}^{169-374} \mathrm{Y} 353 \mathrm{E}\right)$. Stereochemistry was analysed with MolProbity (Chen et al., 2010). Structure superpositions were calculated using the SuperPose server (Maiti et al., 2004) and buried surface areas and residue contacts were obtained using the PISA server (Krissinel \& Henrick, 2007). Illustrations of protein structures were prepared using CCP4mg (McNicholas et al., 2011).

\subsection{Chemical cross-linking of purified SPOP proteins}

Protein was purified by size-exclusion chromatography in PBS and cross-linked by incubation with $6 \mathrm{~m} M N$-hydroxysulfosuccinimide, $6 \mathrm{~m} M$ 1-ethyl-3-(3-dimethylaminopropyl)carbodiimide hydrochloride for $2 \mathrm{~h}$ at $294 \mathrm{~K}$.

\subsection{Atomic force microscopy}

A freshly cleaved mica surface was incubated for $10 \mathrm{~min}$ in $5 \mathrm{mM} \mathrm{MgCl}$, briefly rinsed with deionized water

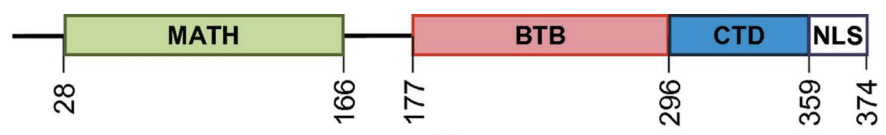

(a)

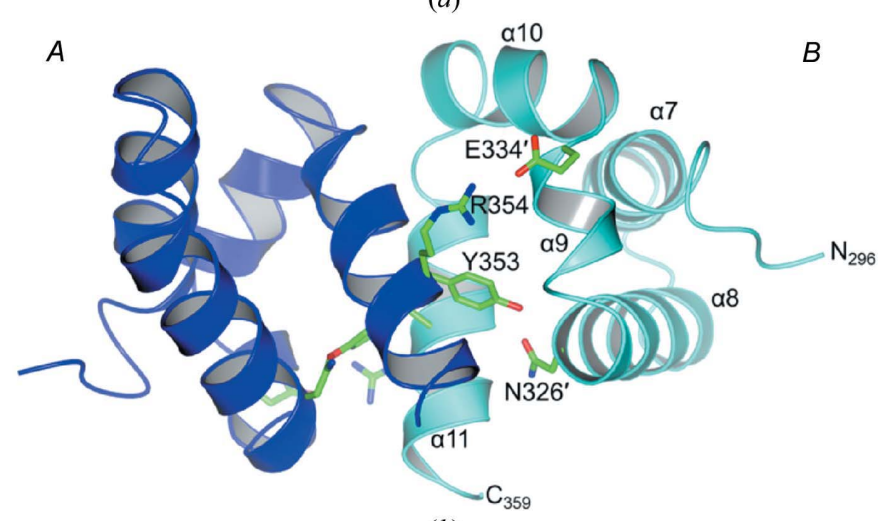

(b)

Figure 1

Crystal structure of the SPOP C-terminal domain (CTD). (a) Domain organization of human SPOP. (b) Cartoon representation of the SPOP CTD dimer. The two chains are coloured blue and cyan. Secondarystructure elements of the $B$ chain and interacting residues of the dimerization interface are indicated; residues of the $B$ chain are indicated with a prime. $\left(18.2 \mathrm{M} \Omega \mathrm{cm}^{-1}\right)$ and dried in a stream of $\mathrm{N}_{2}$ gas; $\mathrm{Mg}^{2+}$ modifies the negatively charged mica surface, thus assisting the binding of SPOP protein, which is also negatively charged at neutral $\mathrm{pH}$. Purified SPOP ${ }^{169-374}$ was diluted to $1 \mu \mathrm{g} \mathrm{ml}^{-1}$ in PBS pH 7.4 supplemented with $5 \mathrm{mM} \mathrm{MgCl}$, and the mica surface was incubated for $30 \mathrm{~min}$ with $50 \mu \mathrm{l}$ of the diluted protein. The disc was gently and briefly washed with deionized water $\left(18.2 \mathrm{M} \Omega \mathrm{cm}^{-1}\right)$ and dried in a stream of $\mathrm{N}_{2}$ gas.

The sample was imaged using a Multimode 8 atomic force microscope (Bruker) using PPP-NCLR probes (Nanosensors; resonant frequency $170 \mathrm{kHz}$ ). Images were acquired in attractive-regime amplitude-modulation tapping mode, operating with small r.m.s. amplitudes of $<0.15 \mathrm{~V}$. The images shown are from two independently purified samples of SPOP protein; each batch was imaged at least three times. Data were analysed and processed using NANOSCOPE ANALYSIS v.1.40r1 (Bruker).

\section{Results}

\subsection{Crystal structure of the SPOP C-terminal domain}

Human SPOP is a 374-residue protein that contains an $\mathrm{N}$-terminal MATH domain (residues 28-166) that recruits substrates and a central BTB domain (residues 177-296) that mediates dimerization and interactions with $\mathrm{Cul} 3$ (Fig. 1a). The SPOP C-terminus is predicted to contain an $\alpha$-helical region (residues 298-359) and a basic nuclear localization signal (NLS; residues 365-374), and it has recently been shown that the C-terminus is involved in dynamic high-order oligomerization (Errington et al., 2012). To analyse the oligomerization properties of the SPOP C-terminus, we expressed SPOP $^{169-374}$, which contains the BTB domain together with the C-terminus, in E. coli. The purified protein eluted as a high-molecular-weight species $(>100 \mathrm{kDa})$ upon sizeexclusion chromatography, and chemical cross-linking of the protein produced high-order oligomers (Supplementary Fig. S1 $a^{\mathbf{1}}$ ). In contrast, the purified BTB domain, SPOP ${ }^{169-296}$, was dimeric, as was a protein that contained an incomplete $\mathrm{C}$-terminus $\left(\mathrm{SPOP}^{169-334}\right)$, suggesting that the $\mathrm{C}$-terminal residues 335-374 are required for high-order oligomerization (Supplementary Fig. S1a). To study the C-terminus of SPOP in isolation from the BTB domain, we purified SPOP $^{270-374}$ L273D L282D L285K, which contains the two Cterminal $\alpha$-helices ( $\alpha 5$ and $\alpha 6$ ) of the BTB domain together with the entire SPOP C-terminus; the solubility of this protein was increased by the mutation of three surface-exposed hydrophobic residues of $\alpha 5$ and $\alpha 6$. SPOP $270-374$ L273D L282D L285K was dimeric when analysed by size-exclusion chromatography and chemical cross-linking (Supplementary Fig. S1b), suggesting that the SPOP C-terminus contains a dimerization interface that is independent of the BTB domain.

To elucidate the mechanism of the high-order oligomerization of SPOP, we determined the X-ray crystal

\footnotetext{
${ }^{\mathbf{1}}$ Supplementary material has been deposited in the IUCr electronic archive (Reference: MH5093). Services for accessing this material are described at the back of the journal.
} 
Table 1

Data-collection and refinement statistics.

Values in parentheses are for the highest resolution shell.

\begin{tabular}{|c|c|c|c|}
\hline & SPOP $^{270-374 ~ L 273 D ~ L 282 D ~ L 285 K, ~ n a t i v e ~}$ & SPOP $^{270-374 ~ L 273 D ~ L 282 D ~ L 285 K ~}$, SeMet & SPOP $^{169-374}$ Y353E , native \\
\hline PDB code & $4 \mathrm{hs} 2$ & & $4 \mathrm{j} 8 \mathrm{z}$ \\
\hline \multicolumn{4}{|l|}{ Crystal parameters } \\
\hline Unit-cell parameters $\left(\AA,^{\circ}\right)$ & $\begin{array}{l}a=57.65, b=57.65, c=102.81 \\
\alpha=90.00, \beta=90.00, \gamma=120.00\end{array}$ & $\begin{array}{l}a=57.58, b=57.58, c=102.81 \\
\quad \alpha=90.00, \beta=90.00, \gamma=120.00\end{array}$ & $\begin{array}{c}a=111.39, b=71.58, c=62.22 \\
\quad \alpha=90.00, \beta=117.21, \gamma=90.00\end{array}$ \\
\hline \multicolumn{4}{|l|}{ Data collection } \\
\hline Resolution (Å) & $51.40-1.53(1.57-1.53)$ & $51.40-2.12(2.18-2.12)$ & $58.02-2.42(2.48-2.42)$ \\
\hline$R_{\text {p.i.m }} \ddagger(\%)$ & $1.7(25.0)$ & $2.9(15.7)$ & 2.7 (21.6) \\
\hline$\langle I / \sigma(I)\rangle$ & $24.2(3.1)$ & $38.4(9.0)$ & $17.2(3.7)$ \\
\hline No. of unique reflections & 16004 (1143) & $6183(437)$ & $16513(1207)$ \\
\hline Multiplicity & $11.6(8.3)$ & $71.5(60.1)$ & $3.5(3.5)$ \\
\hline Completeness (\%) & $99.8(99.9)$ & $100.0(100.0)$ & $98.9(98.1)$ \\
\hline \multicolumn{4}{|l|}{ Refinement } \\
\hline Resolution $(\AA)$ & $27.76-1.53$ & & $58.02-2.42$ \\
\hline No. of protein atoms & 542 & & 2762 \\
\hline No. of water molecules & 62 & & 25 \\
\hline Average $B$ factor $\left(\AA^{2}\right)$ & 16.90 & & 64.28 \\
\hline \multicolumn{4}{|l|}{ Ramachandran analysis $\neq \neq(\%)$} \\
\hline Favoured & 100.0 & & 97.7 \\
\hline Allowed & 0.0 & & 2.3 \\
\hline Disallowed & 0.0 & & 0.0 \\
\hline
\end{tabular}

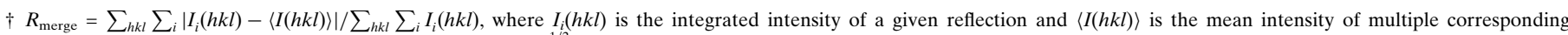

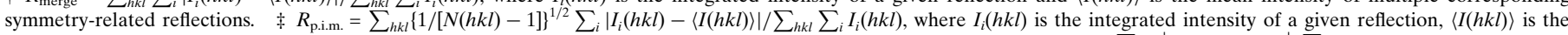

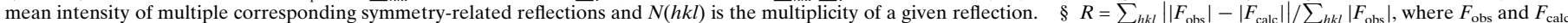

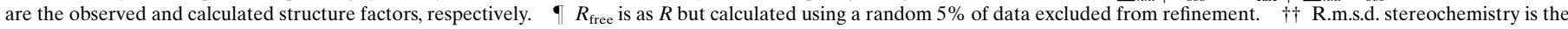
deviation from ideal values. 抹 Ramachandran analysis was carried out using MolProbity (Chen et al., 2010).

structure of the C-terminus at $1.5 \AA$ resolution. The SPOP $^{270-374}$ L273D L282D L285K dimer crystallized with a monomer in the asymmetric unit; the dimer was revealed from crystallographic symmetry (Table 1 , Fig. $1 b$ and Supplementary Fig. S2). No electron density was observed for the residues comprising the predicted nuclear localization signal (residues 360-374) and BTB $\alpha 5-\alpha 6$ (residues 270-295), and they were not built in the model; disordering of $\alpha 5-\alpha 6$ has also been observed in other structures of BTB-domain fragments that lack the BTB N-terminus (for example, KBTBD4; PDB entry 2eqx; RIKEN Structural Genomics/Proteomics Initiative, unpublished work). The SPOP C-terminal domain (CTD; residues 296-359) comprises five $\alpha$-helices, denoted $\alpha 7-\alpha 11$, with $\alpha 11$ residues contributing most of the interactions at the dimerization interface. The dimer interface buries $685 \AA^{2}$ of surface area per monomer and is stabilized by hydrogen-bond interactions between Tyr353 and Asn326' and between Arg354 and Glu334', together with a salt bridge between Arg354 and Glu334'. The dimer interface and the $\alpha 9-\alpha 10-\alpha 11$ fold are stabilized by the burial of hydrophobic residues that include Val349, Ala350, Ala352, Leu356 and Ala357.

To confirm the role of the $\alpha 11$ residues in the high-order oligomerization of SPOP, we purified proteins that contained the entire BTB domain together with various parts of the C-terminus. SPOP ${ }^{169-359}$, which contains an intact CTD but lacks the predicted NLS, eluted as a high-molecular-weight species upon size-exclusion chromatography and the purified protein could be cross-linked into a high-order oligomer; in contrast, SPOP proteins that had truncations in $\alpha 11$ (SPOP $^{169-344}$ and SPOP ${ }^{169-355}$ ) were dimeric (Supplementary Fig. 1c). The high-order oligomerization of SPOP is thus mediated by two independent self-association interfaces: the classic BTB dimerization interface and the dimerization interface of the CTD.

\subsection{Organization of the SPOP BTB and C-terminal domains}

BTB-domain dimers are domain-swapped dimers that have very low dissociation constants $\left(K_{\mathrm{d}}<200 \mathrm{n} M\right.$; Li et al., 1997), whereas oligomerization directed by the SPOP C-terminus is dynamic and dissociable in solution (Errington et al., 2012). In order to obtain protein crystals of the contiguous SPOP BTB and CTD domains in a single molecule, we therefore attempted to disrupt CTD dimerization by point mutation of an interface residue. We mutated Tyr353 to glutamic acid in the context of the SPOP ${ }^{169-374}$ protein that contained an intact BTB and CTD. In contrast to the high-order oligomerization observed with SPOP ${ }^{169-374}$, SPOP ${ }^{169-374}$ Y353E was dimeric when analysed by size-exclusion chromatography, and the purified protein could be cross-linked into a dimer 
(Supplementary Fig. S1d), confirming the role of Tyr353 in CTD dimerization and in high-order oligomerization.

We determined the X-ray crystal structure of $\mathrm{SPOP}^{169-374} \mathrm{Y} 353 \mathrm{E}$ at $2.4 \AA$ resolution (Table 1 and Fig. $2 a$ ). $\mathrm{SPOP}^{169-374} \mathrm{Y} 353 \mathrm{E}$ crystallized as a BTB-mediated dimer; there were no interactions between the CTD residues of neighbouring SPOP molecules in the crystal, consistent with the complete disruption of the CTD dimerization interface in the SPOP Y353E mutant. Residues 177-329 of SPOP ${ }^{169-374}$ Y353E superposed extremely well (r.m.s.d. value of $0.49 \AA$ for $\mathrm{C}^{\alpha}$-atom positions) with a reported SPOP structure (PDB entry $3 \mathrm{htm}$; SPOP residues 177-329; Zhuang et al., 2009) that contains the classic BTB domain ( $\alpha 1-\alpha 6$; residues 177-296) together with the helical hairpin ( $\alpha 7-\alpha 8$; residues $300-327)$ that was dubbed the 3-box (Zhuang et al., 2009). Additionally, residues 300-356 of SPOP ${ }^{169-374}$ Y353E superposed extremely well with SPOP $^{270-374 ~ L 273 D ~ L 282 D ~ L 285 K ~(r . m . s . d . ~ v a l u e ~ o f ~} 0.46 \AA$ for $\mathrm{C}^{\alpha}$ atom positions; Fig. $3 a$ ), suggesting that the relative orientation of the SPOP CTD and BTB domains is inflexible. No electron density was observed for residues $169-176$ and $357-$ 374 of SPOP ${ }^{169-374}$ Y353E , and residues comprising the $\alpha 3-\beta 4$ loop were associated with higher crystallographic $B$ factors, as reported previously (Zhuang et al., 2009; Errington et al., 2012).

The $\alpha 7-\alpha 8$ hairpin of the SPOP CTD interacts with the $\alpha 5-$ $\alpha 6$ helices of the BTB domain to form an antiparallel fourhelix bundle, whereas the CTD helices $\alpha 9-\alpha 10-\alpha 11$ form a distinct motif that lies perpendicular to $\alpha 7-\alpha 8$. The overall fold of the SPOP CTD resembles the N-terminal portion of the 130-residue $\alpha$-helical BACK (BTB and C-terminal Kelch) domain that is found C-terminal to the BTB domain in the Kelch-type Cul3 adaptors (Stogios \& Privé, 2004). The structure of SPOP ${ }^{169-374}$ Y353E superposed well (r.m.s.d value of $2.48 \AA$ for $\mathrm{C}^{\alpha}$-atom positions) with the contiguous BTB and BACK domains of the Kelch-type BTB adaptor KLHL11 (PDB entry 3i3n; Canning et al., 2013), with the relative orientation of the BTB and BACK domains being conserved between the two proteins (Fig. $2 b$ ). The residues preceding $\alpha 1$ of the BTB domain are oriented differently in SPOP and KLHL11, and interact with different regions of the opposite chain in the domain-swapped dimer, as reported previously (Canning et al., 2013; Errington et al., 2012).

\subsection{Model of the high-order SPOP oligomer}

We generated a model of the high-order SPOP oligomer by iterative superposition of the dimeric SPOP ${ }^{169-374}$ Y353E and $\mathrm{SPOP}^{270-374}$ L273D L282D L285K structures that contain the BTBand CTD-interaction interfaces, respectively (Fig. 3a). We also modelled the substrate-recruiting MATH domains using an available SPOP structure (PDB entry 3hqi; Zhuang et al., 2009). The model of the high-order SPOP oligomer depicts alternating BTB and CTD dimers assembled in a left-handed helical manner that reflects the skewed arrangement of the constituent twofold-symmetry axes (Figs. $3 a$ and $3 b$ ). Although the extent of high-order oligomerization in vivo is not known, a full turn of the SPOP helix would contain approximately 16
SPOP chains (eight BTB and eight CTD dimers) and have a pitch of $510 \AA$. The SPOP MATH domains are flexibly tethered to the BTB domain (Zhuang et al., 2009) and are organized in a contiguous array along one face of the oligomer. To confirm the model of the high-order SPOP oligomer, we visualized the SPOP ${ }^{169-374}$ complex using atomic force microscopy (AFM). AFM images depict a linear organization of the subunits of the SPOP oligomer; most of the molecules have a curved or horseshoe shape, consistent with a helical structure that has broken and kinked during the sequential deposition of its subunits onto the grid (Fig. $3 c$ and Supplementary Fig. S3).

We also modelled a high-order oligomer of the SPOP E3 ligase complex that contains $\mathrm{Cul} 3$ and the E2-binding RING protein RBX1 (Fig. 3d). This model was generated by superposition using common regions from available structures: the

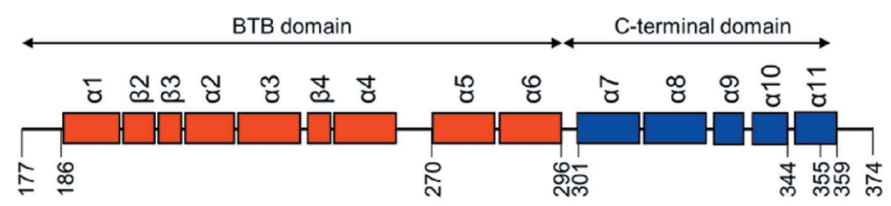

(a)

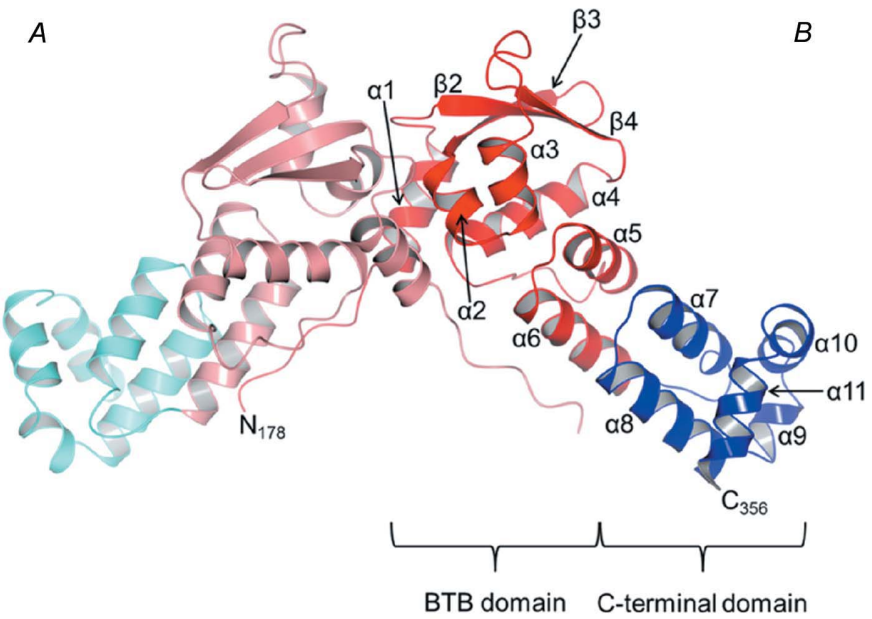

(b)

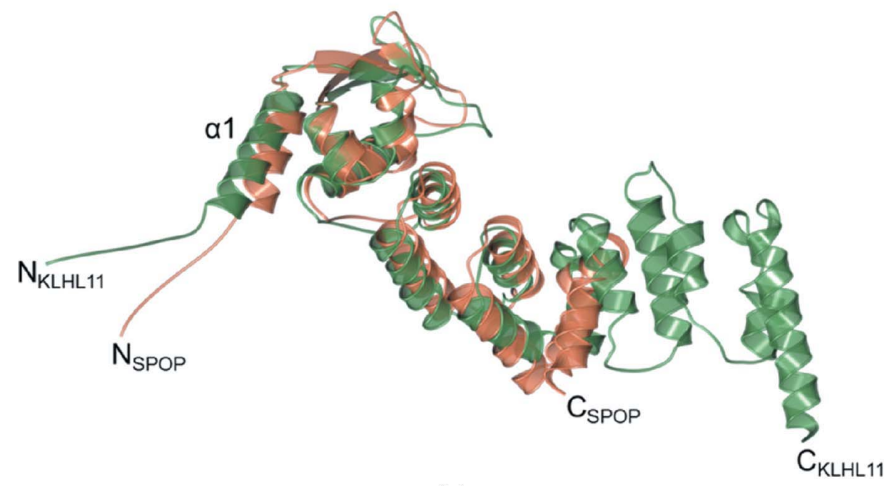

(c)

Figure 2

Organization of the SPOP BTB and C-terminal domains. (a) Crystal structure of the SPOP ${ }^{169-374}$ Y353E dimer. BTB domains are coloured red and pink; CTDs are coloured blue and cyan. Secondary-structure elements of the $B$ chain are indicated. $(b)$ Superposition of SPOP $^{169-374}$ Y353E (orange) with the BTB and BACK domains of KLHL11 (PDB entry 3i3n; green). 
first two cullin repeats were from SPOP ${ }^{\mathrm{BTB}}-\mathrm{Cul} 3$ (PDB entry 4eoz; Errington et al., 2012) and the third cullin repeat and the cullin C-terminal domain together with RBX1 were from Cul1-RBX1 (PDB entry 1ldj; Zheng et al., 2002). The model of the high-order SPOP oligomer is compatible with the recruitment of a Cul3 molecule to each SPOP chain without

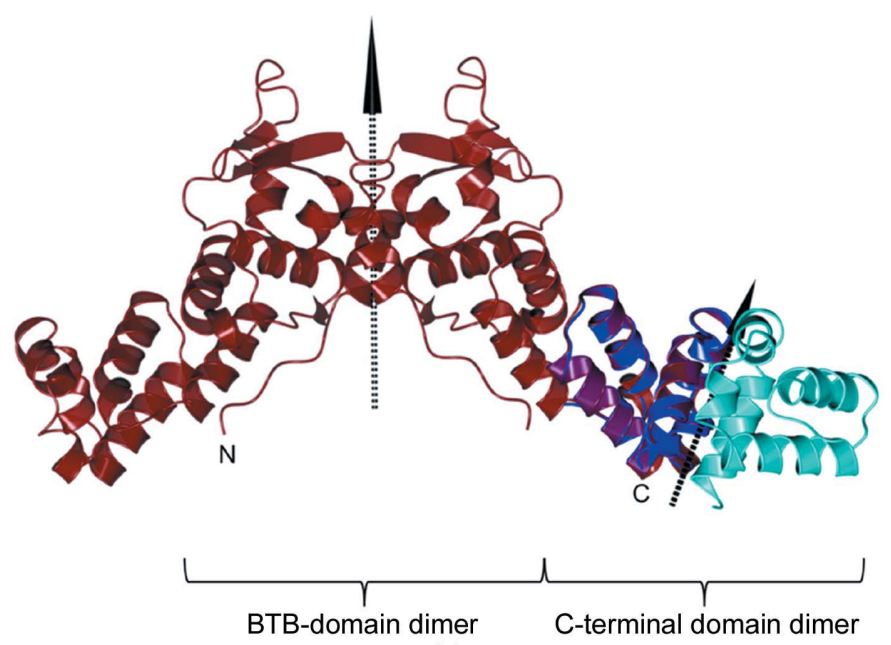

(a)

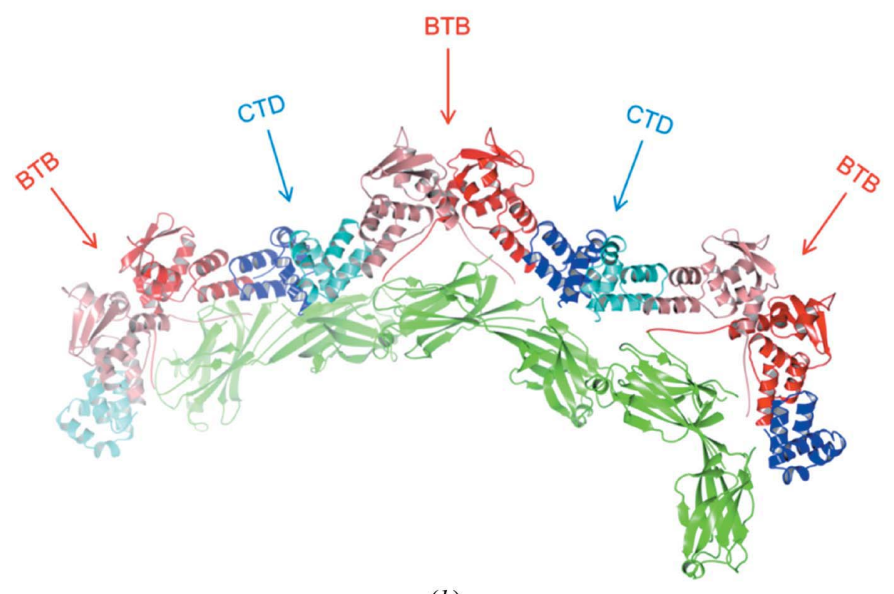

(b)

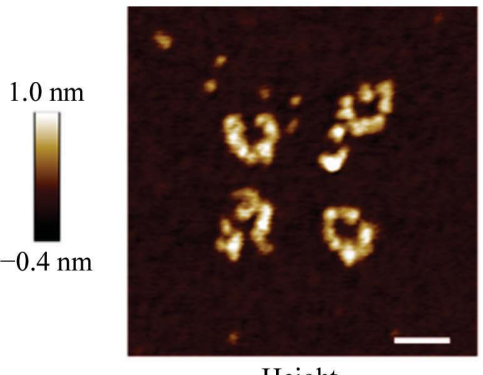

Height

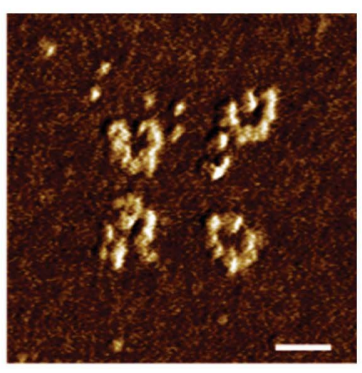

Phase

(c) creating steric clashes. The model suggests that the N-terminus of $\mathrm{Cul3}$ interacts with residues from both the BTB and CTD domains of SPOP, consistent with a recent structure that revealed the interaction of $\mathrm{Cul} 3$ with both the $\mathrm{BTB}$ and BACK domains of KLHL11 (PDB entry 4ap2; Canning et al., 2013); the relative orientation of the $\mathrm{BTB}$ and $\mathrm{Cul} 3$ molecules is identical in the KLHL11-Cul3 and SPOP-Cul3 structures (PDB entries 4ap2 and 4eoz, respectively). The elongated Cul3 molecules extend in arcs away from the SPOP BTB-CTD backbone to form two rows of arms that bend towards the inner curve of the helix. The RBX1 molecules that interact with the $\mathrm{C}$-termini of the cullins to recruit the E2-conjugating enzyme are thus arranged in two rows that flank the central MATH domains on the same face of the assembly.

\section{Discussion}

The model of the high-order SPOP-Cul3-RBX1 oligomer provides insight into the possible mechanisms whereby adaptor oligomerization regulates substrate ubiquitination. The $\alpha$-helical core of the contiguous SPOP CTD and BTB domains forms a rigid framework upon which multiple $\beta$-sheet MATH domains are flexibly tethered along one face. This arrangement of MATH domains could enhance the efficiency and versatility of substrate recruitment by increasing the rate of binding and by facilitating simultaneous interactions with multiple degrons that might be variably positioned within

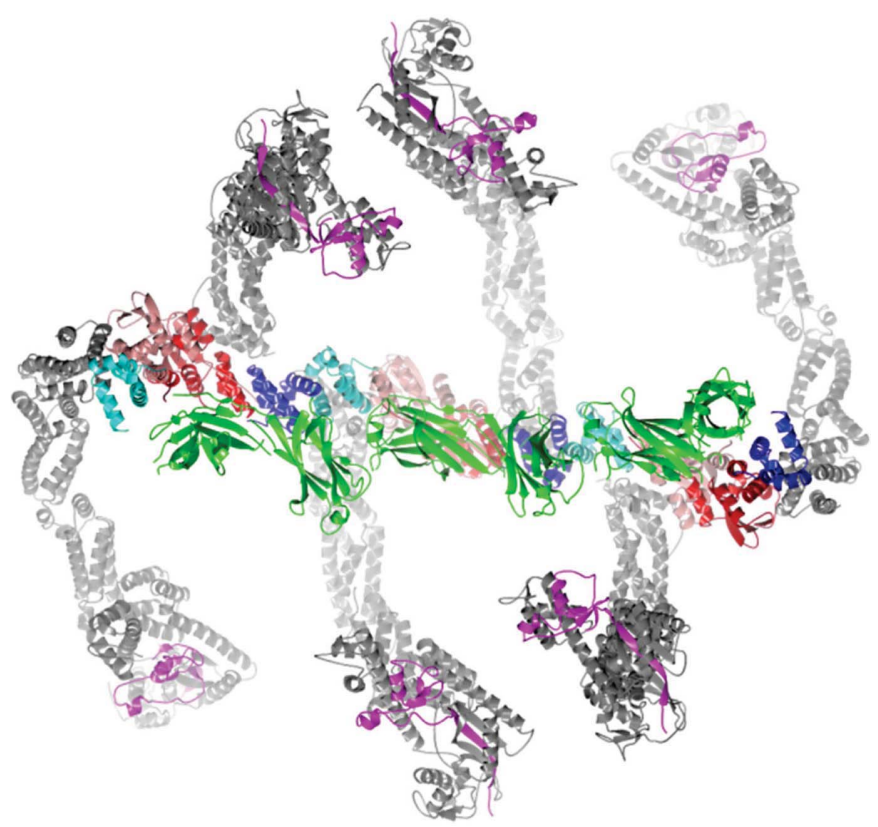

(d)

Figure 3

High-order SPOP oligomers. (a) Superposition of the SPOP CTD dimer (blue and cyan) with the SPOP ${ }^{169-374 ~ Y 353 E}$ dimer (brown). The rotation axes of the CTD and BTB domains are indicated. $(b)$ Model of a high-order SPOP oligomer. The model depicts six SPOP molecules and was generated by iterative superposition of the SPOP CTD dimer with the SPOP ${ }^{169-374}$ Y353E dimer using structural alignment of the common $\alpha 7-\alpha 10$ residues (300-344). BTB domains are coloured red and pink; CTDs are coloured blue and cyan. The SPOP MATH domains (residues 28-166; green) that recruit substrates for ubiquitination were modelled by superposition from the SPOP ${ }^{\mathrm{BTB}+\mathrm{MATH}}$ structure (PDB entry 3 hqi). (c) AFM images of SPOP ${ }^{169-374}$ oligomers. A representative example is shown. The scale bar corresponds to $50 \mathrm{~nm}$. (d) Model of a high-order SPOP-Cul3-RBX1 complex. The SPOP oligomer was used as a template on which Cul3 and RBX1 were modelled. The first two cullin repeats of the Cul3 N-terminal domain were modelled using the structure of SPOP ${ }^{\mathrm{BTB}}$-Cul3 (PDB entry 4eoz); the third cullin repeat, the cullin C-terminal domain and RBX1 were modelled using the structure of Cul1-RBX1 (PDB entry 1ldj). Cullin chains are coloured grey and RBX1 chains are coloured magenta. 
a single substrate molecule; such cooperative multivalent interactions would be particularly important in the recognition of substrates with multiple weak degrons (Zhang et al., 2009; Zhuang et al., 2009). The high-order oligomerization of SPOP would also result in the concentration of multiple E2 conjugating enzyme molecules close to the substrate on the same face of the assembly, thereby increasing the rate of the ubiquitination reaction.

The mechanisms that modulate the oligomeric state of SPOP in vivo remain to be determined, although it is feasible that such regulation might provide a mechanism for the differential ubiquitination of individual substrates according to the number and strength of the degrons that they contain. It has been reported that the SPOP C-terminal region is required for the ubiquitination and degradation of the substrate Daxx in transfected mammalian cells (Kwon et al., 2006), consistent with the importance of high-order oligomerization in vivo. The human genome encodes a closely related SPOP paralogue, SPOPL (Choo et al., 2010), that interacts both with SPOP substrates and with Cul3 in vitro (Errington et al., 2012) and is highly conserved among vertebrates. The BTB domains of SPOP and SPOPL are $87 \%$ identical and the two proteins form BTB-mediated heterodimers in vitro; in contrast, the SPOP and SPOPL CTDs differ, with the SPOPL CTD containing an 18-residue insertion that prevents high-order oligomerization (Errington et al., 2012). SPOPL restricts the high-order oligomerization of SPOP in mixtures of the two proteins in vitro; this results from the formation of BTBmediated hetero-oligomers and it has therefore been speculated that the relative levels of SPOP and SPOPL in vivo might determine the degree of SPOP oligomerization (Errington et al., 2012). The dimerization interface of the SPOP CTD buries a relatively small surface area and can be completely disrupted by the mutation of Tyr353 to glutamic acid; it is therefore highly suited for directing the dynamic high-order association of the more stable BTB-mediated SPOP dimers and it will be relevant to determine whether oligomerization is regulated by the phosphorylation of Tyr353 in vivo.

The SPOP CTD resembles the N-terminal portion of the $\alpha$-helical BACK domain that is found C-terminal to the BTB domain in the Kelch-type Cul3 adaptors. It is predicted that Cul3 interacts with the SPOP CTD in a manner that resembles its interaction with the BACK domain of the Kelch-type adaptor KLHL11, and the relative orientation of the BTB and Cul3 molecules is identical in SPOP-Cul3 and KLHL11-Cul3. It is not known whether the BACK domain mediates highorder oligomerization of the BTB Kelch-type adaptors: the Kelch-type adaptors Keap1 and gigaxonin have been reported as BTB-mediated dimers (Ogura et al., 2010; Zhuang et al., 2009) and crystal structures of BACK domains have not thus far revealed self-interaction interfaces (PDB entries 3hve, 2eqx and 3i3n). Thus, although the BTB-mediated SPOP-Cul3 and KLHL11-Cul3 dimers have a similar architecture, the SPOP CTD may represent a truncated BACK domain that has evolved an additional specialized function in high-order oligomerization; it is feasible that differences in the oligo- merization properties of the individual BTB MATH-type and Kelch-type Cul3 adaptors have evolved alongside differences in the modes of substrate recognition and of regulation.

Protein self-assembly provides both architectural and regulatory roles in biological systems, and high-order oligomerization is a feature of proteins that contain more than one distinct self-association domain. For example, the two independent self-association interfaces of the centriole protein, SAS-6, direct its assembly into a cartwheel structure with ninefold symmetry that forms the basis of the centriolar core (Kitagawa et al., 2011). The high-order oligomerization of BTB-domain proteins has been well documented, although the structural basis for these associations has thus far remained elusive. The model of the high-order SPOP oligomer described here provides a structural basis for the regulation of substrate ubiquitination by adaptor oligomerization; this will be relevant for understanding the control of ubiquitination and may direct the rational design of inhibitors for use in the therapeutic targeting of ubiquitination complexes.

We thank the staff of beamlines I02, I04-1 and I03 at Diamond Light Source for advice on X-ray data collection. This work was funded by Yorkshire Cancer Research (grant LPP037) and a Cancer Research UK studentship to HJC (award C37059/A12514).

\section{References}

Barbieri, C. E. et al. (2012). Nature Genet. 44, 685-689.

Canning, P., Cooper, C. D., Krojer, T., Murray, J. W., Pike, A. C., Chaikuad, A., Keates, T., Thangaratnarajah, C., Hojzan, V., Marsden, B. D., Gileadi, O., Knapp, S., von Delft, F. \& Bullock, A. N. (2013). J. Biol. Chem. 288, 7803-7814.

Choo, K.-B., Chuang, T.-J., Lin, W.-Y., Chang, C.-M., Tsai, Y.-H. \& Huang, C.-J. (2010). Gene, 460, 39-47.

Cohen, S. X., Ben Jelloul, M., Long, F., Vagin, A., Knipscheer, P., Lebbink, J., Sixma, T. K., Lamzin, V. S., Murshudov, G. N. \& Perrakis, A. (2008). Acta Cryst. D64, 49-60.

Chen, V. B., Arendall, W. B., Headd, J. J., Keedy, D. A., Immormino, R. M., Kapral, G. J., Murray, L. W., Richardson, J. S. \& Richardson, D. C. (2010). Acta Cryst. D66, 12-21.

Emsley, P., Lohkamp, B., Scott, W. G. \& Cowtan, K. (2010). Acta Cryst. D66, 486-501.

Errington, W. J., Khan, M. Q., Bueler, S. A., Rubinstein, J. L., Chakrabartty, A. \& Privé, G. G. (2012). Structure, 20, 1141-1153.

Espinás, M. L., Jiménez-García, E., Vaquero, A., Canudas, S., Bernués, J. \& Azorín, F. (1999). J. Biol. Chem. 274, 16461-16469.

Evans, P. (2006). Acta Cryst. D62, 72-82.

Kabsch, W. (2010). Acta Cryst. D66, 125-132.

Kim, B., Nam, H. J., Pyo, K. E., Jang, M. J., Kim, I. S., Kim, D., Boo, K., Lee, S. H., Yoon, J.-B., Baek, S. H. \& Kim, J. H. (2011). Biochem. Biophys. Res. Commun. 415, 720-726.

Kitagawa, D., Vakonakis, I., Olieric, N., Hilbert, M., Keller, D., Olieric, V., Bortfeld, M., Erat, M. C., Flückiger, I., Gönczy, P. \& Steinmetz, M. O. (2011). Cell, 144, 364-375.

Komander, D. \& Rape, M. (2012). Annu. Rev. Biochem. 81, 203-229. Krissinel, E. \& Henrick, K. (2007). J. Mol. Biol. 372, 774-797.

Kwon, J. E., La, M., Oh, K. H., Oh, Y. M., Kim, G. R., Seol, J. H., Baek, S. H., Chiba, T., Tanaka, K., Bang, O. S., Joe, C. O. \& Chung, C. H. (2006). J. Biol. Chem. 281, 12664-12672.

Li, C., Ao, J., Fu, J., Lee, D.-F., Xu, J., Lonard, D. \& O’Malley, B. W. (2011). Oncogene, 30, 4350-4364.

Li, X., Lopez-Guisa, J. M., Ninan, N., Weiner, E. J., Rauscher, F. J. III \& Marmorstein, R. (1997). J. Biol. Chem. 272, 27324-27329. 
Liu, J. et al. (2009). Science, 323, 1218-1222.

Maiti, R., Van Domselaar, G. H., Zhang, H. \& Wishart, D. S. (2004). Nucleic Acids Res. 32, W590-W594.

McCoy, A. J., Grosse-Kunstleve, R. W., Adams, P. D., Winn, M. D., Storoni, L. C. \& Read, R. J. (2007). J. Appl. Cryst. 40, 658-674.

McNicholas, S., Potterton, E., Wilson, K. S. \& Noble, M. E. M. (2011). Acta Cryst. D67, 386-394.

Murshudov, G. N., Skubák, P., Lebedev, A. A., Pannu, N. S., Steiner, R. A., Nicholls, R. A., Winn, M. D., Long, F. \& Vagin, A. A. (2011). Acta Cryst. D67, 355-367.

Nagai, Y., Kojima, T., Muro, Y., Hachiya, T., Nishizawa, Y., Wakabayashi, T. \& Hagiwara, M. (1997). FEBS Lett. 418, 23-26.

Nalepa, G., Rolfe, M. \& Harper, J. W. (2006). Nature Rev. Drug Discov. 5, 596-613.

Ogura, T., Tong, K. I., Mio, K., Maruyama, Y., Kurokawa, H., Sato, C. \& Yamamoto, M. (2010). Proc. Natl Acad. Sci. USA, 107, 28422847.

Petroski, M. D. (2008). BMC Biochem. 9, S7.

Pintard, L., Willems, A. \& Peter, M. (2004). EMBO J. 23, 1681-1687.

Schneider, T. R. \& Sheldrick, G. M. (2002). Acta Cryst. D58, 17721779.
Sheldrick, G. M. (2008). Acta Cryst. A64, 112-122.

Stogios, P. J., Downs, G. S., Jauhal, J. J., Nandra, S. K. \& Privé, G. G. (2005). Genome Biol. 6, R82.

Stogios, P. J. \& Privé, G. G. (2004). Trends Biochem. Sci. 29, 634-637.

Takahashi, I., Kameoka, Y. \& Hashimoto, K. (2002). Biochim. Biophys. Acta, 1591, 63-68.

Winter, G. (2010). J. Appl. Cryst. 43, 186-190.

Zhang, K. Y. J., Cowtan, K. \& Main, P. (1997). Methods Enzymol. 277, 53-64.

Zhang, Q., Shi, Q., Chen, Y., Yue, T., Li, S., Wang, B. \& Jiang, J. (2009). Proc. Natl Acad. Sci. USA, 106, 21191-21196.

Zheng, N., Schulman, B. A., Song, L., Miller, J. J., Jeffrey, P. D., Wang, P., Chu, C., Koepp, D. M., Elledge, S. J., Pagano, M., Conaway, R. C., Conaway, J. W., Harper, J. W. \& Pavletich, N. P. (2002). Nature (London), 416, 703-709.

Zhuang, M., Calabrese, M. F., Liu, J., Waddell, M. B., Nourse, A., Hammel, M., Miller, D. J., Walden, H., Duda, D. M., Seyedin, S. N., Hoggard, T., Harper, J. W., White, K. P. \& Schulman, B. A. (2009). Mol. Cell, 36, 39-50.

Zimmerman, E. S., Schulman, B. A. \& Zheng, N. (2010). Curr. Opin. Struct. Biol. 20, 714-721. 